\title{
Interference Fringes in the Plane Wave Topographic Images of Growth Bands in Si:Ge
}

\author{
K. Wieteska ${ }^{a}$, W. Wierzchowski ${ }^{b}$, W. GraefF ${ }^{c}$, \\ M. LeFELD-SosnowskA ${ }^{d}$ AND M. RegulskA ${ }^{d}$ \\ ${ }^{a}$ Institute of Atomic Energy, 05-400 Otwock-Świerk, Poland \\ ${ }^{b}$ Institute of Electronic Materials Technology \\ Wólczyńska 133, 01-919 Warsaw, Poland \\ ${ }^{c}$ HASYLAB at DESY, Notkestrasse 85, 22603 Hamburg, Germany \\ ${ }^{d}$ Institute of Experimental Physics, University of Warsaw \\ Hoża 69, 00-681 Warsaw, Poland
}

\begin{abstract}
An Si:Ge crystal with approximately $3 \%$ of germanium was studied with strongly collimated short-wavelength monochromatic synchrotron beam (beamline E2 at HASYLAB). The topographs obtained in the asymmetric 224 reflection revealed the presence of interference fringes related to growth bands caused by segregation of germanium. The fringes, observed for the first time, were strongly dependent on the angular setting and it was possible to distinguish at least three systems of fringes. A number of features of the existing strain field, which may be important for the formation of the fringes, was determined using other topographic methods, especially the Bragg-case section topography.
\end{abstract}

PACS numbers: $61.72 . \mathrm{Ff}$

\section{Introduction}

The formation of interference fringes in X-ray topographic images is very interesting both in cognitive and practical aspects. In the cognitive aspect it is interesting for a better understanding of diffraction phenomena and a further development of the diffraction theory. In the practical aspect, it can be used for the determination of the strain fields and the identification of defects existing in the examined crystals. 
A number of papers were already devoted to growth striations arriving in Czochralski grown silicon single crystals (as-grown and annealed) [1,2] or doped with carbon $[3,4]$. The growth striations in quartz were observed and analysed in [5]. Plane wave topography in transmission and/or back reflection geometry, realized with laboratory X-ray sources, were applied in these investigations.

In the present paper we report the observation of interference fringes connected with growth striations in Si:Ge single crystal in back-reflection geometry. The interference fringes related to growth striations in carbon doped Si were observed in transmission geometry by Jenichen and Köhler [3, 4]. The present observation was performed using a very well collimated synchrotron monochromatic beam in Si:Ge crystals containing approximately $3 \%$ of germanium. The practical aspect of the present investigation is connected with the significant technological difficulty in obtaining Si:Ge bulk crystals with uniform Ge concentration, caused by the high segregation coefficient of germanium in silicon [6-8]. For this reason the study of growth bands may provide data for improving the growth technology. In the actual case, the monochromatic beam investigation was completed by the use of other topographic methods and rocking curve measurements.

\section{Experimental}

The investigated sample was cut off perpendicularly to the [111] growth axis from an Si:Ge crystal with $3 \%$ of germanium. The crystal was grown at the Institute of Electronic Materials Technology (Warsaw) by the Czochralski technique in argon flow with a growth velocity of $2 \mu \mathrm{m} / \mathrm{s}$. The sample was a $400 \mu \mathrm{m}$ thick plate, mechanically and chemically polished on both sides.

The observation of the interference fringes caused by striations was performed at the monochromatic beam station E2 at HASYLAB. A highly angular collimated beam with a wavelength of $0.11 \mathrm{~nm}$ was obtained using a piezoelectrically stabilised double crystal monochromator with successive 333 and 511 reflections from silicon crystals. The asymmetric 224 reflection with low glancing angle was used. Together with taking topographs, rocking curves were recorded using the beam limited to a small area close to $50 \times 50 \mu \mathrm{m}^{2}$. Some other investigations were performed in 333 symmetrical reflection, where the natural FWHM is approximately twice narrower.

The sample was systematically studied with the number of other X-ray topographic methods employing both conventional and synchrotron sources of X-ray radiation. The investigation included, in particular, Lang methods (in transmission and reflection) and white beam projection and section topography. The transmission Lang topographs were obtained in symmetrical $\overline{22} 4$ reflection, a reflection one in highly asymmetric $551(\theta-\varphi)$ both of Mo $K_{\alpha_{1}}$ radiation. The synchrotron white beam radiation (SWBR) back-reflection topographs were taken with low glancing angle of $4^{\circ}$ and in the case of section topography a fine $5 \mu \mathrm{m}$ slit was used. 


\section{Results and discussion}

A characteristic feature of an $\mathrm{Si}: \mathrm{Ge}$ crystal with a moderate concentration of germanium is the presence of growth striations connected with the segregation of germanium. The striations were dominating in the topographic images obtained with all the applied methods. Two Lang topographs of the studied sample, being a quarter of the whole wafer, are shown in Figs. 1a and b. The images of the striations are regularly circular close to the crystal edge and less regular close to the centre of the wafer, where a kind of striation - free "core" is observed. The better understanding of the Lang image is provided by SWBR Bragg case projection topograph shown in Fig. 2a. The Lang transmission topographs reveal mainly the contrasts corresponding to the outcrops of the striations on the surfaces, which may be better distinguished in the SWBR Bragg case projection topographs. The last provides practically only the images of striation outcrops on the examined surface. Finally the Bragg case section topography (Fig. 2b) provides the image of striations in the plane formed by narrow incident beam inside the crystal $[9,10]$.

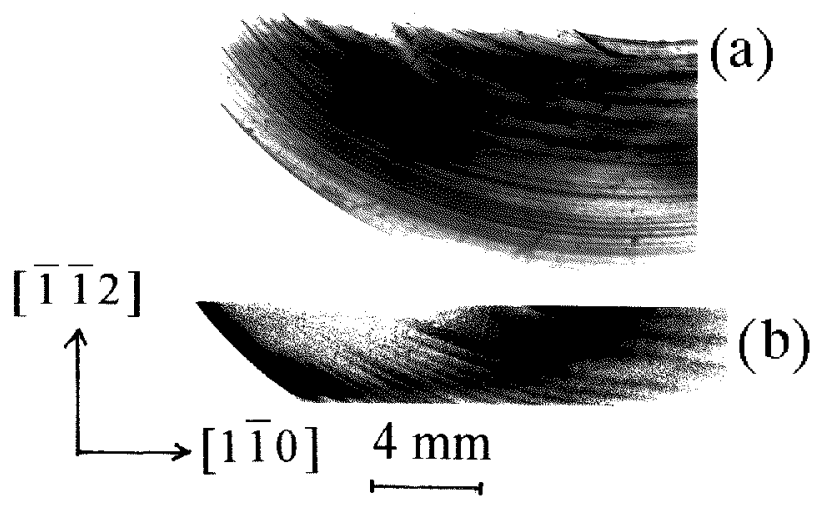

Fig. 1. (a) Projection transmission topograph taken in $\overline{22} 4$ reflection of Mo $K_{\alpha_{1}}$ radiation, (b) projection reflection topograph taken in $551 \theta-\varphi$ reflection of Mo $K_{\alpha_{1}}$ radiation.

The same sample studied in 224 reflection of very well collimated synchrotron monochromatic beam with a wavelength of $0.11 \mathrm{~nm}$ provided images very rich in interference fringes, shown representatively in Figs. 3a and b. The image of these fringes was different for two angular settings corresponding to topographs $3 \mathrm{a}$ and $\mathrm{b}$. It may be noticed that the topographs exhibit some kinds of interference fringes. One characteristic kind are the fringes parallel to the striations while some other systems form wavy patterns with interference maxima crossing the first one. A corresponding experimental rocking curve is shown in Fig. 3c. In this case the illuminated area covered at least two stripes being probably the reason of broadening and formation of two maxima. 


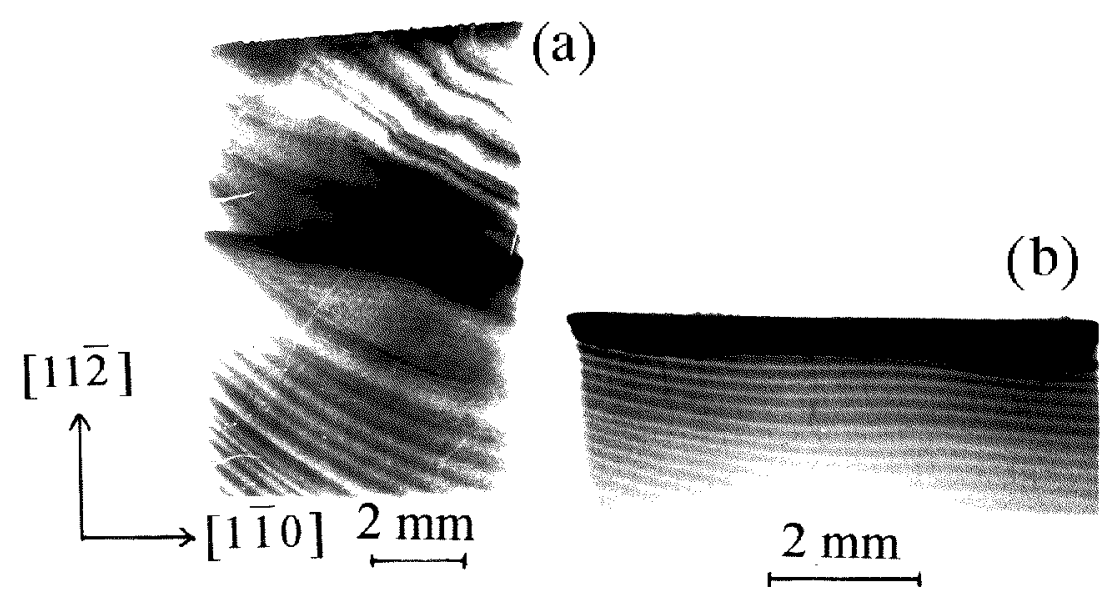

Fig. 2. Synchrotron white beam radiation back-reflection topographs; 224 reflection, $\lambda=0.045 \mathrm{~nm}$; (a) projection topograph with superimposed section topograph, (b) section topograph.

(a)
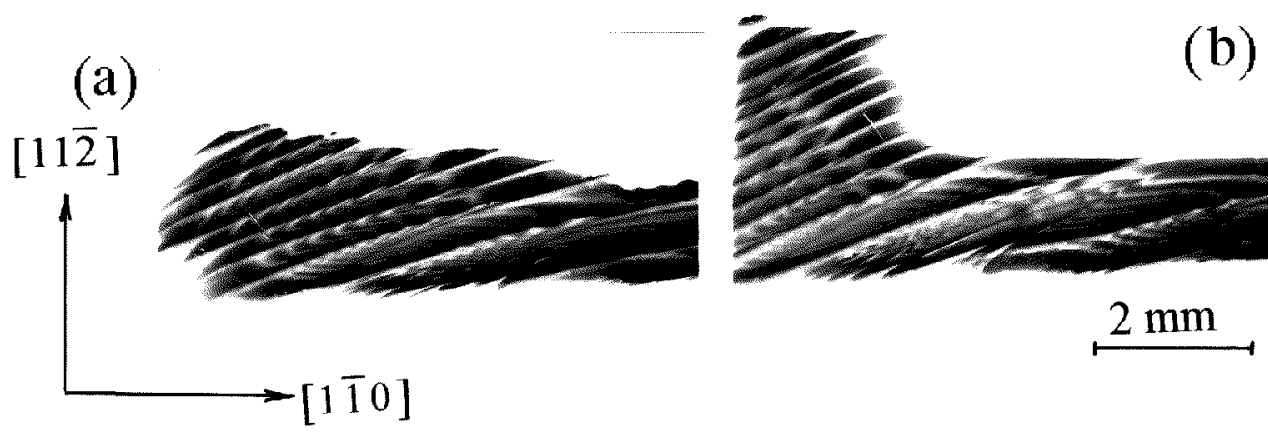

(b)

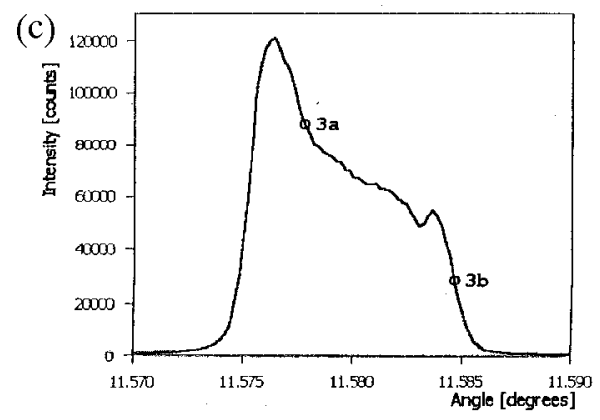

Fig. 3. Synchrotron monochromatic plane wave back-reflection topographs; 224 reflection, $\lambda=0.11 \mathrm{~nm}$; (a) angular crystal position marked as 3a in (c); (b) angular crystal position marked as $3 \mathrm{~b}$ in (c); (c) rocking curve with marked positions $3 \mathrm{a}$ and $3 \mathrm{~b}$.

Contrary to the results in the 224 reflection, the investigation in 333 reflection, where the rocking curve was much narrower, did not reveal any fringes. In 
this case the topographs were strongly disturbed by the effects of strains causing reproduction of only narrow area of the sample. The presently obtained fringes were also very sensitive to the angular collimation, which could be easily spoiled by greater heating of the monochromator.

Important information about the strain field connected with the striation and responsible for the formation of interference fringes was provided by the Bragg-case section topograph recorded at large film-to-crystal distance shown in Fig. 4. It may be noticed that the upper edge of the Bragg-case section topograph forms a characteristic saw-like pattern, which corresponds to the lattice deformation, caused by germanium segregation. The range of the angular misorientation forming the saw was evaluated as approximately $15^{\prime \prime}$. The upper parts of the teeth correspond to the greater glancing angle and this situation should occur for oblique reflecting planes in the compressed germanium rich regions. It may be also expected that the "saw" should be a good approximation of the germanium distribution.

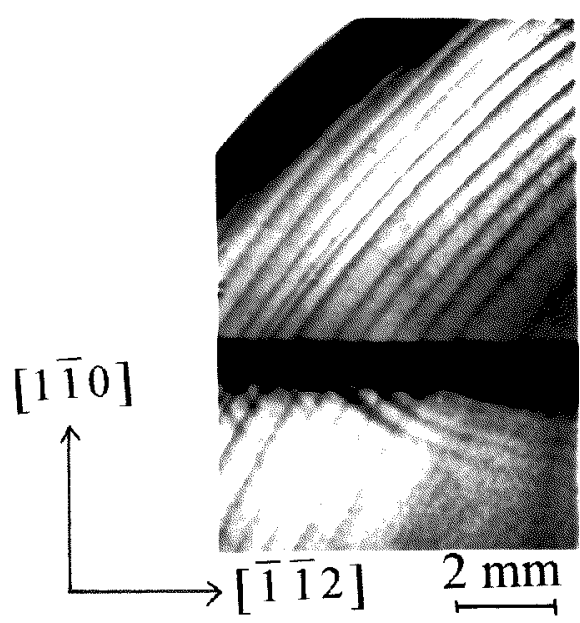

(b)

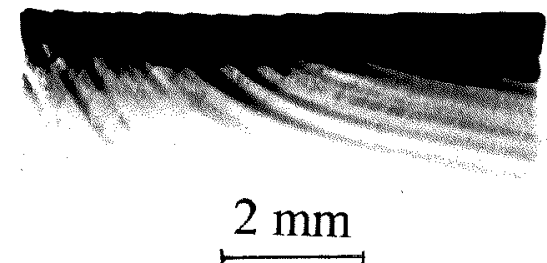

Fig. 4. SWBR back-reflection topographs, 264 reflection, $\lambda=0.033 \mathrm{~nm}$; (a) projection topograph with superimposed section topograph, (b) section topograph - the upper part of the image forms the characteristic saw.

The characteristic black contrasts in the Bragg-case section topograph visible in Fig. 2a, which often forms double stripes, are connected with the upper and lower parts of each tooth where the strain gradient is the highest. It may be found that the interference fringes in the 224 plane wave topographs are present mainly in the regions between teeth maxima corresponding to the right less steep slope.

The exact analysis of the observed fringes seems to be very difficult in view of the complicated character of deformation field caused both by surface strain relaxation and its parabolic or less regular shape inside the crystal. The proba- 
ble interpretation of the fringes parallel to the striations is the decomposition of the wave fields, formed in the Bragg case diffraction process, by mostly deformed surfaces corresponding to shorter steeper edges of the teeth. After this decomposition the wave fields are recovered in the Bragg case similarly as in the case of dislocation images discussed by Bedyńska [11]. It is also much probable that the other systems of fringes are connected with weaker strain gradients present in the regions of less steeper slope. The character of this gradient is not well recognizable in the saw pattern.

As it was already mentioned, the important factor for fringe formation seems to be a relatively large width of the rocking curve of 224 reflection close to $4^{\prime \prime}$. That causes smaller sensitivity to the strains. On the other hand, the great width of the rocking curve reduces the effects of angular divergence of the probe beam on the diffraction picture.

\section{Conclusions}

The present paper reports an observation and discussion of interference fringes, composed of multiple systems, formed at striations present in Si:Ge crystal. The observation of the fringes was enabled by the use of reflection with relatively wide rocking curve and a relatively large distance between the striations. Some of the features of the deformation field of the crystal causing formation of the fringes on topographs, especially the existence of regions with high and low strain gradient and its location inside the crystal were revealed by means of SWBR Bragg-case section topography and other topographic investigations.

\section{References}

[1] J. Kubĕna, V. Holý, Czech. J. Phys. B 34, 950 (1984).

[2] J. Kubĕna, V. Holý, Czech. J. Phys. B 35, 1007 (1985)

[3] B. Jenichen, R. Köhler, Phys. Status Solidi A 65, 535 (1981).

[4] B. Jenichen, R. Köhler, Phys. Status Solidi A 65, 245 (1981).

[5] H.-J. Bernhardt, J. Härtwig, V. Lerche, Prog. Crystal Growth Charact. 24, 1 (1992).

[6] I. Yonenaga, A. Matsui, S. Tozawa, K. Sumino, T. Fukuda, J. Cryst. Growth 154, 275 (1995)

[7] N.V. Abrosimov, S.N. Rossolenko, V. Alex, A. Gerhardt, W. Schröder, J. Cryst. Growth 166, 657 (1996).

[8] M. Lefeld-Sosnowska, Z. Grygoruk, K. Wokulska, J. Błażewicz, J. Phys. D, Appl. Phys. 34, A 144 (2001).

[9] K. Wieteska, W. Wierzchowski, W. Graeff, M. Lefeld-Sosnowska, HASYLAB Jahresbericht 2000, p. 891.

[10] K. Wieteska, W. Wierzchowski, W. Graeff, M. Lefeld-Sosnowska, M. Regulska, Mater. Sci. Eng. B, in press.

[11] T. Bedyńska, Phys. Status Solidi A 18, 147 (1973). 